\title{
THE REGULARITY OF THE LOCALLY INTEGRABLE AND CONTINUOUS SOLUTIONS OF NONLINEAR FUNCTIONAL EQUATIONS
}

\author{
BY \\ HALINA ŚWIATAK
}

\begin{abstract}
The purpose of this paper is to show a general method which allows one to find all the continuous (and sometimes also all the locally integrable) solutions of functional equations by considering solutions of class $C^{m}$. One can do it if one is assured that all the continuous (or all the locally integrable) solutions of a given equation are functions of class $C^{m}$ or $C^{\infty}$. Such a property is characteristic for the solutions $f: R^{n} \rightarrow R$ of the equations (*) $k$ where $x \in R^{n}, t \in R^{r}, n>1, r>1$ and where the functions $\phi_{i}: R^{n+r} \rightarrow$ $R^{n}, \lambda_{j}: R^{n} \rightarrow R^{n}, a_{i}: R^{n+r} \rightarrow R, b: R^{n+r} \rightarrow R, F: R^{n+s} \rightarrow R$ satisfy some regularity assumptions and the assumptions which guarantee that an equation obtained by differentiating (*) and fixing $t$ is of constant strength, hypoelliptic at a point $x_{0}$. A general theorem, concerning the regularity of the continuous and locally integrable solutions $f$ of (*), is formulated and proved by the reduction to the corresponding problem for the distributional solutions of linear partial differential equations.
\end{abstract}

1. Introduction. It often happens in functional equations that very weak regularity assumptions allow one to prove that all the solutions which satisfy these weak assumptions are functions of class $C^{\infty}$.

There exist numerous examples of functional equations, with unknown functions depending on one variable, for which the continuity at single points implies continuity, differentiability, or even analyticity, everywhere. Such equations will not be considered here.

Many results of this type are known also in the theory of partial differential equations. For instance all solutions of an elliptic differential equation with analytical coefficients and analytical forcing term are analytic functions; all solutions of an elliptic differential equation with $C^{\infty}$ coefficients and $C^{\infty}$ forcing term are functions of class $C^{\infty}$; all solutions of an elliptic differential equation of order $1 \leqslant m$ with coefficients and forcing term of class $C^{m}$ are functions of class $C^{m}$.

Received by the editors July 23, 1974.

AMS (MOS) subject classifications (1970). Primary 39A15, 39A30, 46F99; Secondary 26A54, 26A93, 35G99, 35H05, 35J15. 
(Similar statements are true for a larger class of differential equations, namely, for differential equations of constant strength hypoelliptic at a point.) Hence it follows immediately that if a functional equation implies, e.g., an elliptic differential equation of order $m$ with $C^{\infty}$ coefficients and $C^{\infty}$ forcing term, then all its solutions of class $C^{m}$ are $C^{\infty}$ functions. This information can help to solve such an equation in the class $C^{m}$ since some resulting differential equations of higher order may then be considered.

However, when investigating a functional equation, one is interested not only in its solutions of class $C^{m}$, but also in its continuous or less regular solutions.

It will be shown that if the equations considered are such as to imply linear partial differential equations of order $m$, of constant strength, hypoelliptic at an $x_{0}$, with $C^{\infty}$ coefficients and $C^{\infty}$ forcing term, then not only solutions of class $C^{m}$ but also all continuous solutions are functions of class $C^{\infty}$. Therefore, to solve such an equation in the class of continuous functions, one may reduce it to differential equations and find differentiable solutions, being assured that no other continuous solutions exist.

Theorems presented in this paper allow one to recognize functional equations for which this method can be applied.

The paper investigates equations of the form

$$
\sum_{i=1}^{k} a_{i}(x, t) f\left(\phi_{i}(x, t)\right)=F\left(x, f\left(\lambda_{1}(x)\right), \ldots, f\left(\lambda_{s}(x)\right)\right)+b(x, t),
$$

where $x \in R^{n}, t \in \Omega \subset R^{r}, n>1, r \geqslant 1, \phi_{i}: R^{n} \times \Omega \rightarrow R^{n}, \lambda_{j}: R^{n} \rightarrow R^{n}$, $a_{i}: R^{n} \times \Omega \rightarrow R, b: R^{n} \times \Omega \rightarrow R, F: R^{n+s} \rightarrow R$.

Proofs of the regularity of the integrable solutions of similar classes of functional equations are well-known when $n=r=1$ (see e.g. J. Acźel [1, pp. 183209]). All these proofs apply classical analysis methods which fail if $n>1$.

In this paper a distributional method (different than that one used by the author in [19]) is used.

Other distributional methods were applied by I. Fenyö [6], [7], [8] to solve some functional equations with unknown functions depending on one variable, by A. M. Garsia [13] and by A. Friedman and W. Littman [12] in connection with mean value equations, by G. Choquet and J. Deny [4] to prove some properties of harmonic functions and by J. A. Baker [3] in connection with a functional equation being an analogue to the wave equation.

Equations (1.1) include the well-known mean value equations

$$
\sum_{i=1}^{k} \mu_{i} f\left(x+\alpha_{i} t\right)=f(x) \quad \text { with } \sum_{i=1}^{k} \mu_{i}=1
$$


and, therefore, have a small overlap with the generalized mean value equations

$$
\int f(x+r t) d \mu(t)=0,
$$

where $\mu$ is a finite complex Borel measure supported in the closed unit ball in $R^{n}$. Different properties of equations (1.2) and (1.3) were studied, e.g., in [8][14], [17], [28]. (For more references concerning (1.3) see [28].) It is not the aim of this paper to generalize those results although, e.g., in the case $\phi_{i}(x, t)=x+\phi_{i}(t)$ some such generalizations are possible (see B. Lawruk and H. Swiatak [16] and H. Swiatak [25]).

The regularity of the continuous and locally integrable solutions $f$ of (1.1) is the only property which will be studied here. One should add, for the sake of completeness, that a much stronger result concerning the regularity of the function $f$ satisfying (1.2) was obtained by M. A. McKiernan [17]. Also the paper [21] dealing with a special case of (1.1), namely, $\phi_{i}(x, t)=x+\phi_{i}(t)$ and $F\left(x, u_{1}, \ldots, u_{s}\right) \equiv 0$ proves some stronger results. (The papers concerning (1.3) assume the continuity of $f$.)

2. Notation and definitions.

$$
\begin{gathered}
x, y, \xi, \eta, 0 \in R^{n} ; t, \alpha \in R^{r}, \\
p=\left(p_{1}, \ldots, p_{n}\right), \quad \text { where the } p_{i} \text { are integers } \geqslant 0, \\
q=\left(q_{1}, \ldots, q_{r}\right), \quad \text { where the } q_{i} \text { are integers } \geqslant 0, \\
|p|=p_{1}+\cdots+p_{n}, \quad|q|=q_{1}+\cdots+q_{r}, \\
D^{p}=D_{x}^{p}=\frac{\partial^{|p|}}{\partial x_{1}^{p_{1}} \cdots \partial x_{n}^{p_{n}}}, \quad D^{q}=D_{t}^{q}=\frac{\partial^{|q|}}{\partial t_{1}^{q_{1}} \cdots \partial t_{r}^{q_{r}}}, \\
\operatorname{supp} \psi=\overline{\{x: \psi(x) \neq 0\},} \\
D=\left\{\psi: \psi: R^{n} \rightarrow R, \psi \in C^{\infty} \text { in } R^{n}, \text { supp } \psi \text { is compact }\right\} .
\end{gathered}
$$

We say that a sequence $\psi_{\nu} \rightarrow 0$ in $D$ if there exists a compact set $E \subset R^{n}$ such that for every $\nu$, supp $\psi_{\nu} \subset E$ and $D^{p} \psi_{\nu} \rightarrow 0$ uniformly for each $p$.

The operator $T: \psi \in D \rightarrow(T, \psi) \in R$ is called a distribution if it inear, and continuous in the following sense: If $\psi_{\nu} \rightarrow 0$ in $D,\left(T, \psi_{\nu}\right) \rightarrow 0$ in $R$.

The set of all distributions in $R^{n}$ will be denoted by $D^{\prime} .(T, \psi)$ will be denoted also by $(T(x), \psi(x))_{x}$.

Let $\Omega \subset R^{r}$, and assume $\theta: R^{n} \times \Omega \rightarrow R$. Then for each fixed $t \in \Omega$, $\Theta_{t}(x)=\theta(x, t)$ defines a function $\Theta_{t}: R^{n} \rightarrow R$. In analogy to the above, if $\Theta_{t} \in D$ and $T \in D^{\prime}$, we may define

$$
\gamma(t)=\left(T, \Theta_{t}\right)=(T(x), \theta(x, t))_{x} .
$$


We will also need notation for functions from $R^{n} \times \Omega \rightarrow R^{n}$ :

$$
\begin{gathered}
\Phi(x, t)=\left(\Phi_{1}(x, t), \ldots, \Phi_{n}(x, t)\right), \\
D_{t}^{q} \Phi(x, t)=\left(D_{t}^{q} \Phi_{1}(x, t), \ldots, D_{t}^{q} \Phi_{n}(x, t)\right), \\
\Phi: R^{n} \times \Omega \rightarrow R^{n}, \Phi_{j}: R^{n} \times \Omega \rightarrow R, \\
\phi_{i}(x, t)=\left(\phi_{i 1}(x, t), \ldots, \phi_{i n}(x, t)\right), \\
D_{t}^{q} \phi_{i}(x, t)=\left(D_{t}^{q} \phi_{i 1}(x, t), \ldots, D_{t}^{q} \phi_{i n}(x, t)\right), \\
\phi_{i}: R^{n} \times \Omega \rightarrow R^{n}, \phi_{i j}: R^{n} \times \Omega \rightarrow R,
\end{gathered}
$$

where $j=1, \ldots, n$ and $i=1, \ldots, k$.

If $p=\left(p_{1}, \ldots, p_{n}\right)$ is an $n$-tuple of nonnegative integers and $\xi=\left(\xi_{1}\right.$, $\left.\ldots, \xi_{n}\right) \in R^{n}$, we define $\xi^{p}$ by $\xi^{p}=\xi_{1}^{p_{1}} \cdots \xi_{n}^{p_{n}}$.

We say that $\Phi \in C^{m}$ in $R^{n} \times \Omega$, where $\Omega$ is an open set contained in $R^{r}$, if $\Phi_{j} \in C^{m}$ in $R^{n} \times \Omega$ for $j=1, \ldots, n$. We shall often write $\Phi(x, t) \in C^{m}$ instead of $\Phi \in C^{m}$.

We write $\Phi(x, t) \in C^{\bar{m}}$ in $R^{n}$ for each fixed $t \in \Omega$ if the functions $\Phi_{t}: R^{n}$ $\rightarrow R^{n}$, defined by $\Phi_{t}: x \mapsto \Phi_{t}(x)=\Phi(x, t)$, are functions of class $C^{\bar{m}}$ in $R^{n}$ for all parameters $t \in \Omega$.

If $a: R^{n} \times \Omega \rightarrow R$ and if the functions $a_{t}$, defined as $x \mapsto a_{t}(x)=a(x, t)$, are functions of class $C^{\bar{m}}$ in $R^{n}$ for all parameters $t \in \Omega$, we write $a(x, t) \in C^{\bar{m}}$ in $R^{n}$ for every fixed $t \in \Omega$.

A distribution $T \in D^{\prime}$ is said to be a function of class $C^{\infty}$ if there exists a function $f \in C^{\infty}$ in $R^{n}$ such that

$$
(T, \psi)=\int_{R^{n}} f(x) \psi(x) d x \text { for each } \psi \in D .
$$

The definitions of ellipticity and hypoellipticity as well as the definition of a differential operator of constant strength can be found, e.g., in [15, pp. 177; $99,100 ; 171,35,10]$.

3. Basic theorems. This chapter contains some well-known theorems which will be applied later. All of these can be found in [15], [18] and [27] but it is convenient to list them and to refer to this chapter in the proofs.

THEOREM 3.1. If $T \in D^{\prime}$, then the equality

$$
\left(D^{p} T, \psi\right)=(-1)^{|p|}\left(T, D^{p} \psi\right) \text { for each } \psi \in D
$$

defines the new distribution $D^{p} T \in D^{\prime}$.

THEOREM 3.2. If $T \in D^{\prime}, a \in C^{\infty}$ in $R^{n}$, then the equality 


$$
(a T, \psi)=(T, a \psi) \text { for each } \psi \in D
$$

defines the new distribution $a T \in D^{\prime}$.

THEOREM 3.3. If $T \in D^{\prime}$, and the mapping $x \mapsto \Phi(x)$ is a diffeomorphism, then the equality

$$
(T(\Phi(x)), \psi(x))_{x}=\left(T(y), \psi\left(\Phi^{-1}(y)\right)\left|\operatorname{det} \frac{\partial \Phi_{\nu}^{-1}}{\partial y_{\mu}}\right|\right)_{y} \text { for each } \psi \in D
$$

defines the new distribution $T \circ \Phi=T(\Phi) \in D^{\prime}$.

Theorem 3.4. If $T \in D^{\prime}, \theta(x, t) \in C^{m}$ in $R^{n} \times G$, where $G$ is an open set in $R^{r}, \Theta_{t} \in C^{\infty}$ in $R^{n}$ for every fixed $t \in G$, and if there exists a compact set $E \subset R^{n}$ such that supp $\Theta_{t} \subset E$ for each $t \in G$, then $\gamma(t)=(T(x), \theta(x, t))_{x}$ is of class $C^{m}$ in $G$ and

$$
D^{q} \gamma(t)=\left(T(x), D_{t}^{q} \theta(x, y)\right)_{x} \text { in } G
$$

for $|q| \leqslant m$.

THEOREM 3.5. For every $T \in D^{\prime}$ there exists a sequence of functions $f_{\nu} \in$ $C^{\infty}$ in $R^{n}$ such that

$$
\left(f_{\nu}, \psi\right) \rightarrow(T, \psi) \text { when } \nu \rightarrow \infty
$$

for each $\psi \in D$.

THEOREM 3.6. If the differential operator

$$
P(x, D)=\sum_{|p|<m} a_{p}(x) D^{p}
$$

is of constant strength in $R^{n}$, hypoelliptic at an $x_{0}$, and if $a_{p} \in C^{\infty}, b \in C^{\infty}$ in $R^{n}$, then every distributional solution $T$ of the equation

$$
\sum_{|p|<m} a_{p}(x) D^{p} T(x)=b(x)
$$

is a function of class $C^{\infty}$ in $R^{n}$ (see Hörmander [15, p. 176]).

THEOREM 3.7.A. Every elliptic differential operator $P(x, D)=$ $\Sigma_{|p|<m} a_{p}(x) D^{p}$ is of constant strength (see Hörmander $[15$, p. 177]).

THEOREM 3.7.B. Every elliptic differential operator $P(x, D)=$ $\Sigma_{|p|<m} a_{p}(x) D^{p}$ is hypoelliptic for every fixed $x_{0}$.

THEOREM 3.7.C. Every distributional solution $T$ of the elliptic differential equation $\Sigma_{|p|<m} a_{p}(x) D^{p} T(x)=b(x)$, where $a_{p} \in C^{\infty}$ and $b \in C^{\infty}$ in $R^{n}$, is a function of class $C^{\infty}$. 
THEOREM 3.8. Every distributional solution $T$ of the hypoelliptic differential equation

$$
\sum_{|p|<m} a_{p} D^{p} T(x)=b(x),
$$

where $b \in C^{\infty}$ in $R^{n}$, is a function of class $C^{\infty}$.

4. Preliminary results. To prove some theorems on the regularity of the continuous and locally integrable solutions $f$ of equations (1.1) one needs the following lemma:

LEMMA 4.1. If $T \in D^{\prime}, a(x, t) \in C^{\infty}$ in $R^{n}$ for every fixed $t$ from an open set $\Omega \subset R^{r}, a(x, t) \in C^{m}$ in $R^{n} \times \Omega, x \mapsto \Phi(x, t)$ is a diffeomorphism in $R^{n}$ for every fixed $t \in \Omega, \Phi(x, t)$ and its inverse $\Phi^{-1}(y, t)$ are functions of class $C^{m}$ in $R^{n} \times \Omega$, then

$$
D^{q}(a(x, t) T(\Phi(x, t)), \psi(x))_{x}=\left(D_{t}^{q}(a(x, t) T(\Phi(x, t))), \psi(x)\right)_{x}
$$

for $|q| \leqslant m$ and for each $\psi \in D$.

In the special case, where $a(x, t)=a(t)$ and $\Phi(x, t)=x+\Phi(t)$, the proof presented below can be considerably shortened (see H. Swiatak [19, pp. 15, 16]). However, the method of [19] cannot be so easily applied in more complicated cases and seems to be useless if $\Phi(x, t) \neq x+\Phi(t)$.

Before we prove Lemma 4.1, we shall establish some other lemmas which simplify the proof.

LEMMA 4.2. If $f_{\nu} \in C^{\infty}$ in $R^{n}$ for each $\nu, T \in D^{\prime}, x \mapsto \Phi(x, t)$ is a diffeomorphism in $R^{n}$ for every fixed $t \in \Omega \subset R^{r}$, and if $\left(f_{\nu}, \psi\right) \rightarrow(T, \psi)$ when $\nu \rightarrow \infty$ for each $\psi \in D$, then

$$
\left(f_{\nu}(\Phi(x, t)), \psi(x)\right)_{x} \rightarrow(T(\Phi(x, t)), \psi(x))_{x} \quad \text { when } \nu \rightarrow \infty
$$

for each $\psi \in D$.

Proof. Let

$$
K(y, t)=\left|\operatorname{det} \partial \Phi_{\sigma}^{-1}(y, t) / \partial y_{\mu}\right|
$$

(This notation will be used also in the other lemmas of this chapter.)

It follows from Theorem 3.3 and from our assumptions that

$$
\begin{aligned}
\left(f_{\nu}(\Phi(x, t)),\right. & \psi(x))_{x}=\left(f_{\nu}(y), \psi\left(\Phi^{-1}(y, t)\right) K(y, t)\right)_{y} \\
& \rightarrow\left(T(y), \psi\left(\Phi^{-1}(y, t)\right) K(y, t)\right)_{y}=(T(\Phi(x, t)), \psi(x))_{x} .
\end{aligned}
$$

(It is easy to see that the functions $\Psi_{t}: y \mapsto \Psi_{t}(y)=\psi\left(\Phi^{-1}(y, t)\right) K(y, t)$ are elements of the space $D$.) 
LEMmA 4.3. If $f_{\nu} \in C^{\infty}$ in $R^{n}$ for each $\nu, T \in D^{\prime}, x \mapsto \Phi(x, t)$ is a diffeomorphism in $R^{n}$ for every fixed $t \in \Omega \subset R^{r}$, and if $\left(f_{\nu}, \psi\right) \rightarrow(T, \psi)$ when $\nu \rightarrow \infty$ for each $\psi \in D$, then

$$
\left(\frac{\partial}{\partial \Phi_{j}} f_{\nu}(\Phi(x, t)), \psi(x)\right)_{x} \rightarrow\left(\frac{\partial}{\partial \Phi_{j}} T(\Phi(x, t)), \psi(x)\right)_{x} \text { when } \nu \rightarrow \infty
$$

for each $\psi \in D$.

Proof. In view of Theorems 3.3 and 3.1 and in view of our assumptions

$$
\begin{aligned}
\left(\frac{\partial}{\partial \Phi_{j}} f_{\nu}(\Phi(x, t)), \psi(x)\right)_{x}=\left(\frac{\partial}{\partial y_{i}} f_{\nu}(y), \psi\left(\Phi^{-1}(y, t)\right) K(y, t)\right)_{y} \\
\quad=-\left(f_{\nu}(y), \frac{\partial}{\partial y_{j}}\left(\psi\left(\Phi^{-1}(y, t)\right) K(y, t)\right)\right)_{y} \\
\rightarrow-\left(T(y), \frac{\partial}{\partial y_{j}}\left(\psi\left(\Phi^{-1}(y, t)\right) K(y, t)\right)\right)_{y} \\
\quad=\left(\frac{\partial}{\partial y_{j}} T(y), \psi\left(\Phi^{-1}(y, t)\right) K(y, t)\right)_{y}=\left(\frac{\partial}{\partial \Phi_{j}} T(\Phi(x, t)), \psi(x)\right)_{x}
\end{aligned}
$$

when $\nu \rightarrow \infty$ for each $\psi \in D$.

LEMMA 4.4. If $f \in C^{\infty}$ in $R^{n}, a(x, t) \in C^{\infty}$ in $R^{n}$ for every fixed $t$ from an open set $\Omega \subset R^{r}, a(x, t) \in C^{m}$ in $R^{n} \times \Omega, x \mapsto y=\Phi(x, t)$ is a diffeomorphism in $R^{n}$ for every fixed $t \in \Omega, \Phi(x, t) \in C^{m}$ in $R^{n} \times \Omega$, then

$$
D^{q}(a(x, t) f(\Phi(x, t)), \psi(x))_{x}=\left(D_{t}^{q}(a(x, t) f(\Phi(x, t))), \psi(x)\right)_{x}
$$

for $|q| \leqslant m$ and for each $\psi \in D$.

Proof. Notice that $A(x, t)=a(x, t) f(\Phi(x, t)) \psi(x)$ is a function of class $C^{m}$ in $R^{n} \times \Omega$ and therefore

$$
\begin{aligned}
D^{q}(a(x, t) f(\Phi(x, t)), \psi(x))_{x} & =D^{q} \int a(x, t) f(\Phi(x, t)) \psi(x) d x \\
\int D_{t}^{q}(a(x, t) f(\Phi(x, t))) \psi(x) d x & =\left(D_{t}^{q}(a(x, t) f(\Phi(x, t))), \psi(x)\right)_{x}
\end{aligned}
$$

for $|q| \leqslant m$ and for each $\psi \in D$.

LEMMA 4.5. If $T \in D^{\prime}, a(x, t) \in C^{\infty}$ in $R^{n}$ for every fixed $t$ from an open set $\Omega \subset R^{r}, a(x, t) \in C^{1}$ in $R^{n} \times \Omega, x \mapsto y=\Phi(x, t)$ is a diffeomorphism in $R^{n}$ for every fixed $t \in \Omega, \Phi(x, t)$ and its inverse $\Phi^{-1}(y, t)$ are functions of class $C^{1}$ in $R^{n} \times \Omega$, then

$$
D^{q}(a(x, t) T(\Phi(x, t)), \psi(x))_{x}=\left(D_{t}^{q}(a(x, t) T(\Phi(x, t))), \psi(x)\right)_{x}
$$

for $|q|=1$ and for each $\psi \in D$. 
Proof. By Theorem 3.2 and Theorem 3.3 we obtain

where

$$
\begin{aligned}
D^{q}(a(x, t) T(\Phi(x, t)), \psi(x))_{x} & =D^{q}(T(\Phi(x, t)), a(x, t) \psi(x))_{x} \\
& =D^{q}(T(y), \theta(y, t))_{y},
\end{aligned}
$$

$$
\theta(y, t)=a\left(\Phi^{-1}(y, t), t\right) \psi\left(\Phi^{-1}(y, t)\right) K(y, t) .
$$

We have to establish that one can apply the formula given in Theorem 3.4 for $t \in \Omega$. To do this it is sufficient to show that the assumptions of Theorem 3.4 are satisfied for $t$ from every bounded and open set $G \subset \Omega$.

It is easy to see that the function

$$
\Theta_{t}: y \mapsto \Theta_{t}(y)=\theta(y, t)=a\left(\Phi^{-1}(y, t), t\right) \psi\left(\Phi^{-1}(y, t)\right) K(y, t)
$$

is a function of class $C^{\infty}$ in $R^{n}$ for every fixed $t \in \Omega$ and that $\theta(y, t) \in C^{1}$ in $R^{n} \times \Omega$. This holds also for any $G \subset \Omega$. Let us fix an arbitrary open and bounded set $G \subset \Omega$.

We are going to show that for each $\psi \in D$ and for each compact set $F$ such that $G \subset F \subset \Omega$, there exists a compact set $E \subset R^{n}(E$ dependent on $\psi$ and on F) such that

$$
\bigcup_{t \in F} \operatorname{supp} \Theta_{t} \subset E
$$

Notice that

$$
\operatorname{supp} \Theta_{t}=\operatorname{supp} \Psi_{t} \cap \operatorname{supp} K_{t} \subset \operatorname{supp} \Psi_{t},
$$

where

$$
\begin{aligned}
\Psi_{t}: y \mapsto \Psi_{t}(y) & =\psi\left(\Phi^{-1}(y, t)\right), \\
K_{t}: y \mapsto K_{t}(y) & =a\left(\Phi^{-1}(y, t), t\right) K(y, t) .
\end{aligned}
$$

Therefore, to prove (4.4), it is enough to show that the set $\bigcup_{t \in F} \operatorname{supp} \Psi_{t}$ is compact and take this set as $E$.

The compactness of the set $\bigcup_{t \in F}$ supp $\Psi_{t}$ becomes obvious when one observes that this set is the image of the compact set supp $\psi \times F$ under the continuous mapping $\Phi$. In fact,

$$
\bigcup_{t \in F} \operatorname{supp} \Psi_{t}=\bigcup_{t \in F} \Phi_{t}(\operatorname{supp} \psi)=\Phi(\operatorname{supp} \psi, F)
$$

where $\Phi_{t}(x)$ denotes $\Phi(x, t)$ with a fixed $t$ and

$$
\Phi(\text { supp } \psi, F)=\{y: y=\Phi(x, t),(x, t) \in \operatorname{supp} \psi \times F\} .
$$

It is known that the image of a compact set under a continuous map is 
compact and this finishes the proof of the fact that the formula of Theorem 3.4 can be applied.

Applying this formula one obtains

$$
D^{q}(T(y), \theta(y, t))_{y}=\left(T(y), D_{t}^{q} \theta(y, t)\right)_{y},
$$

where $|q|=1$ and $\theta(y, t)$ is given by (4.3).

In view of Theorem 3.5, there exists a sequence of functions $f_{\nu} \in C^{\infty}$ in $R^{n}$ such that $\left(f_{\nu}, \psi\right) \rightarrow(T, \psi)$ when $\nu \rightarrow \infty$ for each $\psi \in D$. In particular, it is so for the functions $y \mapsto D_{t}^{q} \theta(y, t) .\left({ }^{1}\right)$ Therefore

$$
\left(T(y), D_{t}^{q} \theta(y, t)\right)_{y}=\lim _{\nu \rightarrow \infty}\left(f_{\nu}(y), D_{t}^{q} \theta(y, t)\right)_{y}
$$

and applying once again Theorem 3.4, one obtains

$$
\lim _{\nu \rightarrow \infty}\left(f_{\nu}(y), D_{t}^{q} \theta(y, t)\right)_{y}=\lim _{\nu \rightarrow \infty} D^{q}\left(f_{\nu}(y), \theta(y, t)\right)_{y} .
$$

Taking into account (4.3) and applying Theorems 3.3 and 3.2 yields

$$
\begin{aligned}
\left(f_{\nu}(y), \theta(y, t)\right)_{y} & =\left(f_{\nu}(y), a\left(\Phi^{-1}(y, t), t\right) \psi\left(\Phi^{-1}(y, t)\right) K(y, t)\right)_{y} \\
& =\left(f_{\nu}(\Phi(x, t)), a(x, t) \psi(x)\right)_{x}=\left(a(x, t) f_{\nu}(\Phi(x, t)), \psi(x)\right)_{x} .
\end{aligned}
$$

Now, (4.7) can be written as

$$
\lim _{\nu \rightarrow \infty}\left(f_{\nu}(y), D_{t}^{q} \theta(y, t)\right)_{y}=\lim _{\nu \rightarrow \infty} D^{q}\left(a(x, t) f_{\nu}(\Phi(x, t)), \psi(x)\right)_{x} .
$$

Applying Lemma 4.4 and taking into account the assumption $|q|=1$, one obtains

$$
\begin{aligned}
D^{q}\left(a(x, t) f_{\nu}(\Phi(x, t)), \psi(x)\right)_{x}=\left(D_{t}^{q}\left(a(x, t) f_{\nu}(\Phi(x, t))\right), \psi(x)\right)_{x} \\
=\left(D_{t}^{q} a(x, t) f_{\nu}(\Phi(x, t))+a(x, t) \sum_{j=1}^{n} D_{t}^{q} \Phi_{j}(x, t) \frac{\partial}{\partial \Phi_{j}} f_{\nu}(\Phi(x, t)), \psi(x)\right)_{x} \\
=\left(D_{t}^{q} a(x, t) f_{\nu}(\Phi(x, t)), \psi(x)\right)_{x} \\
\quad+\sum_{j=1}^{n}\left(a(x, t) D_{t}^{q_{j}}(x, t) \frac{\partial}{\partial \Phi_{j}} f_{\nu}(\Phi(x, t)), \psi(x)\right)_{x}
\end{aligned}
$$

(1) Our assumptions imply immediately that $D_{t}^{q} \theta(y, t) \in C^{\infty}$ in $R^{n}$ for every fixed $t \in \Omega$. The support of the function $(y, t) \mapsto D_{t}^{q} \theta(y, t)$ is contained in the support of the function $(y, t) \mapsto \theta(y, t)$ and therefore the support of the function $y \rightarrow D_{t}^{q} \theta(y, t)$ with a fixed $t$ is contained in the support of the function $y \mapsto \theta(y, t)$ with the same fixed $t$. Since this last support is compact, the first one has to be also compact. (It is a closed subset of a compact set in $R^{n}$ and its compactness is obvious.) However, we do not need to prove that $y \mapsto D_{t}^{q} \theta(y, t)$ is an element of $D$ for any fixed $t \in \Omega$ since such a proof is already contained in the proof of Theorem 3.3. 
In view of Theorem 3.2, the last equality can be written as $D^{q}\left(a(x, t) f_{\nu}(\Phi(x, t)), \psi(x)\right)_{x}=\left(f_{\nu}(\Phi(x, t)), D_{t}^{q} a(x, t) \psi(x)\right)_{x}$

$$
+\sum_{j=1}^{n}\left(\frac{\partial}{\partial \Phi_{j}} f_{\nu}(\Phi(x, t)), a(x, t) D_{t}^{q} \Phi_{j}(x, t) \psi(x)\right)_{x} .
$$

Substituting this into (4.8) and applying Lemmas 4.2 and 4.3 yields

$$
\begin{aligned}
\lim _{\nu \rightarrow \infty}\left(f_{\nu}(y), D_{t}^{q} \theta(y, t)\right)_{y}= & \left(T(\Phi(x, t)), D_{t}^{q} a(x, t) \psi(x)\right)_{x} \\
& +\sum_{j=1}^{n}\left(\frac{\partial}{\partial \Phi_{j}} T(\Phi(x, t)), a(x, t) D_{t}^{q} \Phi_{j}(x, t) \psi(x)\right)_{x} .
\end{aligned}
$$

By Theorem 3.2 and by the definition of a distribution one obtains

$$
\begin{aligned}
\lim _{\nu \rightarrow \infty}\left(f_{\nu}(y), D_{t}^{q} \theta(y, t)\right)_{y}=\left(D_{t}^{q} a(x, t) T(\Phi(x, t)), \psi(x)\right)_{x} \\
\quad+\left(a(x, t) \sum_{j=1}^{n} D_{t}^{q} \Phi_{j}(x, t) \frac{\partial}{\partial \Phi_{j}} T(\Phi(x, t)), \psi(x)\right)_{x} \\
\quad=\left(D_{t}^{q} a(x, t) T(\Phi(x, t))+a(x, t) \sum_{j=1}^{n} D_{t}^{q} \Phi_{j}(x, t) \frac{\partial}{\partial \Phi_{j}} T(\Phi(x, t)), \psi(x)\right)_{x}
\end{aligned}
$$

Since $|q|=1$, the last equality can be written as

$$
\lim _{\nu \rightarrow \infty}\left(f_{\nu}(y), D_{t}^{q} \theta(y, t)\right)_{y}=\left(D_{t}^{q}(a(x, t) T(\Phi(x, t))), \psi(x)\right)_{x},
$$

and taking into account (4.6), (4.5) and (4.2), one obtains the equality

$$
D^{q}(a(x, t) T(\Phi(x, t)), \psi(x))_{x}=\left(D_{t}^{q}(a(x, t) T(\Phi(x, t))), \psi(x)\right)_{x}
$$

which finishes the proof.

Proof of Lemma 4.1. It follows from Lemma 4.5 that Lemma 4.1 is true for $m=1$.

Suppose that (4.1) holds also for $m-1$ and let $\left|q^{*}\right|=m-1,\left|q^{* *}\right|=1$.

Then, in particular,

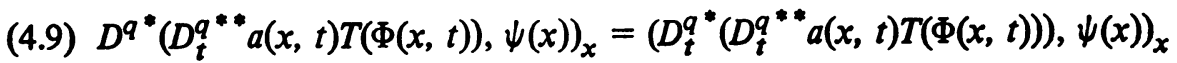

and

$$
\begin{aligned}
& D^{q *}\left(a(x, t) D_{t}^{q * *} \Phi_{j}(x, t) \frac{\partial}{\partial \Phi_{j}} T(\Phi(x, t)), \psi(x)\right)_{x} \\
& =\left(D_{t}^{q^{*}}\left(a(x, t) D_{t}^{q^{* *}} \Phi_{j}(x, t) \frac{\partial}{\partial \Phi_{j}} T(\Phi(x, t))\right), \psi(x)\right)_{x}
\end{aligned}
$$

for $j=1, \ldots, n$ and for each $\psi \in D$ since, in view of our assumptions, 
$D_{t}^{q * *} a(x, t) \in C^{\infty}$ and $a(x, t) D_{t}^{q^{* *}} \Phi_{j}(x, t) \in C^{\infty}$ in $R^{n}$ for every fixed $t \in \Omega \subset$ $R^{r}$ and since $D_{t}^{q^{* *}} a(x, t) \in C^{m-1}$ and $a(x, t) D_{t}^{q^{* *}} \Phi_{j}(x, t) \in C^{m-1}$ in $R^{n} \times \Omega$.

Any $r$-tuple $q$ such that $|q|=m$ can be written as $q=q^{*}+q^{* *}$, where $\left|q^{*}\right|=m-1$ and $\left|q^{* *}\right|=1$.

Therefore, applying Lemma 4.5 and taking into account (4.9), (4.10) and the definition of a distribution, one obtains

$$
\begin{aligned}
D^{q}(a(x, t) T(\Phi(x, t)), \psi(x))_{x}=D^{q^{*}} D^{q^{* *}}(a(x, t) T(\Phi(x, t)), \psi(x))_{x} \\
=D^{q^{*}}\left(D_{t}^{q^{* *}}(a(x, t) T(\Phi(x, t))), \psi(x)\right)_{x} \\
=D^{q^{*}}\left(D_{t}^{q^{* *}} a(x, t) T(\Phi(x, t))\right. \\
\left.\quad \quad+a(x, t) \sum_{j=1}^{n} D_{t}^{q^{* *}} \Phi_{j}(x, t) \frac{\partial}{\partial \Phi_{j}} T(\Phi(x, t)), \psi(x)\right)_{x} \\
=D^{q^{*}}\left(D_{t}^{q^{* *}} a(x, t) T(\Phi(x, t)), \psi(x)\right)_{x} \\
+\sum_{j=1}^{n} D^{q^{*}}\left(a(x, t) D_{t}^{q^{* *}} \Phi_{j}(x, t) \frac{\partial}{\partial \Phi_{j}} T(\Phi(x, t)), \psi(x)\right)_{x} \\
=\left(D_{t}^{q^{*}}\left(D_{t}^{q^{* *}} a(x, t) T(\Phi(x, t))\right), \psi(x)\right)_{x} \\
+\sum_{j=1}^{n}\left(D_{t}^{q^{*}}\left(a(x, t) D_{t}^{q^{* *}} \Phi_{j}(x, t) \frac{\partial}{\partial \Phi_{j}} T(\Phi(x, t))\right), \psi(x)\right)_{x} \\
=\left(D _ { t } ^ { q * } \left(D_{t}^{q^{* *}} a(x, t) T(\Phi(x, t))\right.\right. \\
\left.\left.\quad+a(x, t) \sum_{j=1}^{n} D_{t}^{q^{* *}} \Phi_{j}(x, t) \frac{\partial}{\partial \Phi_{j}} T(\Phi(x, t))\right), \psi(x)\right)_{x} \\
=\left(D_{t}^{q^{*}} D_{t}^{q^{* *}}(a(x, t) T(\Phi(x, t))), \psi(x)\right)_{x} \\
=\left(D_{t}^{q}(a(x, t) T(\Phi(x, t))), \psi(x)\right)_{x}
\end{aligned}
$$

for each $\psi \in D$.

5. General regularity criterion. The purpose of this chapter is to prove a general theorem concerning the regularity of the continuous and locally integrable solutions $f$ of equations (1.1).

THEOREM 5.1. Suppose that

$1^{\circ} a_{i}(x, t) \in C^{\infty}$ and $b(x, t) \in C^{\infty}$ in $R^{n}$ for every fixed $t$ from an open set $\Omega \subset R^{r}, i=1, \ldots, k$, $2^{\circ} a_{i}(x, t) \in C^{m}$ and $b(x, t) \in C^{m}$ in $R^{n} \times \Omega, i=1, \ldots, k$, 
$3^{\circ}$ the mappings $x \mapsto y=\phi_{i}(x, t)$ are diffeomorphisms in $R^{n}$ for every fixed $t \in \Omega, i=1, \ldots, k$,

$4^{\circ} \phi_{i}(x, t) \in C^{m}$ and its inverse $\phi_{i}^{-1}(y, t) \in C^{m}$ in $R^{n} \times \Omega, i=1, \ldots, k$,

$5^{\circ} F\left(x, u_{1}, \ldots, u_{s}\right)$ is continuous in $R^{n+s}$,

$6^{\circ} \lambda_{j}(x)$ are continuous in $R^{n}, j=1, \ldots, s$,

$7^{\circ}$ there exists an $\alpha \in \Omega$ such that $\phi_{i}(x, \alpha) \equiv x$ for $i=1, \ldots, k$,

$8^{\circ}$ there exists an r-tuple $q(|q| \leqslant m)$ such that the equation

$$
\left.D_{t}^{q}\left(\sum_{i=1}^{k} a_{i}(x, t) f\left(\phi_{i}(x, t)\right)\right)\right|_{t=\alpha}=0
$$

with the left-hand side obtained by formal differentiation of the left-hand side of equation (1.1) and by setting next $t=\alpha$ is of constant strength, hypoelliptic at an $x_{0}$.

Then every continuous solution $f$ of equation (1.1) has to be a function of class $C^{\infty}$ and every locally integrable solution $f$ has to be a function of class $C^{\infty}$ almost everywhere.

Proof. Notice first that every continuous function which is equal almost everywhere to a function of class $C^{\infty}$, is also a function of class $C^{\infty}$. In fact, two continuous functions can be equal almost everywhere only if they are identical.

Since all the continuous functions are locally integrable, it is sufficient to prove that every locally integrable solution $f$ of equation (1.1) has to be equal to a function of class $C^{\infty}$ almost everywhere.

It is known that every locally integrable function $f$ can be identified with the distribution $f$ defined by the equality

$$
(f, \psi)=\int_{R^{n}} f(x) \psi(x) d x
$$

for each $\psi \in D$.

Similarly the function $\left.x \mapsto F\left(x, f\left(\lambda_{1}(x)\right), \ldots, f \lambda_{s}(x)\right)\right)$ which is locally integrable in view of $5^{\circ}, 6^{\circ}$, and the assumption on the local integrability of the function $f$ can be identified with the distribution $T_{F}$ defined by

$$
\left(T_{F}, \psi\right)=\int_{R^{n}} F\left(x, f\left(\lambda_{1}(x)\right), \ldots, f\left(\lambda_{s}(x)\right)\right) \psi(x) d x
$$

for each $\psi \in D$.

Analogously

$$
(b(x, t), \psi(x))_{x}=\int_{R^{n}} b(x, t) \psi(x) d x
$$

for each $\psi \in D$ and 


$$
\left(a_{i}(x, t) f\left(\phi_{i}(x, t)\right), \psi(x)\right)_{x}=\int_{R^{n}} a_{i}(x, t) f\left(\phi_{i}(x, t)\right) \psi(x) d x
$$

for each $\psi \in D(i=1, \ldots, k)$ define the distributions for any fixed $t \in \Omega$.

Equation (1.1) implies

$$
\begin{aligned}
\sum_{i=1}^{k} \int_{R^{n}} a_{i}(x, t) f\left(\phi_{i}(x, t)\right) \psi(x) d x & \\
& =\int_{R^{n}} F\left(x, f\left(\lambda_{1}(x)\right), \ldots, f\left(\lambda_{s}(x)\right)\right) \psi(x) d x+\int_{R^{n}} b(x, t) \psi(x) d x
\end{aligned}
$$

for each $\psi \in D$.

In view of (5.3)-(5.5) this can be written as

$$
\sum_{i=1}^{k}\left(a_{i}(x, t) f\left(\phi_{i}(x, t)\right), \psi(x)\right)_{x}=\left(T_{F}, \psi\right)+(b(x, t), \psi(x))_{x}
$$

for each $\psi \in D$.

It is easy to see that

$$
\gamma_{i}(t, \psi)=\left(a_{i}(x, t) f\left(\phi_{i}(x, t)\right), \psi(x)\right)_{x}, \quad i=1, \ldots, k,
$$

and

$$
\tilde{\gamma}(t, \psi)=(b(x, t), \psi(x))_{x}
$$

satisfy the assumptions of Lemma 4.1 and therefore the derivatives $D^{q} \gamma_{i}(t, \psi)$ $(i=1, \ldots, k)$ and $D^{q} \tilde{\gamma}(t, \psi)$, where $|q| \leqslant m$, exist and are given by

$$
D^{q} \gamma_{i}(t, \psi)=\left(D_{t}^{q}\left(a_{i}(x, t) f\left(\phi_{i}(x, t)\right)\right), \psi(x)\right)_{x}, \quad i=1, \ldots, k,
$$

and

$$
D^{q} \tilde{\gamma}(t, \psi)=\left(D_{t}^{q} b(x, t), \psi(x)\right)_{x}
$$

for $|q| \leqslant m$.

Since $\left(T_{F}, \psi\right)$ does not depend on $t, D^{q}\left(T_{F}, \psi\right)=0$ and differentiating (5.6) yields

$$
\sum_{i=1}^{k}\left(D_{t}^{q}\left(a_{i}(x, t) f\left(\phi_{i}(x, t)\right)\right), \psi(x)\right)_{x}=\left(D_{t}^{q} b(x, t), \psi(x)\right)_{x}
$$

for each $\psi \in D$, and for $|q| \leqslant m$.

Applying well-known formulas on partial differentiation we can write (5.7) in the form

$$
\sum_{i=1}^{k} \sum_{|p| \leqslant|q|}\left(c_{p i}(x, t) D_{\phi_{i}}^{p} f\left(\phi_{i}(x, t)\right), \psi(x)\right)_{x}=\left(D_{t}^{q} b(x, t), \psi(x)\right)_{x}
$$


for each $\psi \in D$ and for $|q| \leqslant m$. Here

$$
D_{\phi_{i}}^{p} f\left(\phi_{i}(x, t)\right)=\left.D_{y}^{p} f(y)\right|_{y=\phi_{i}(x, t)}
$$

and the coefficients $c_{p i}(x, t)$ depend on $D_{t}^{\bar{q}} a_{i}(x, t)$ and $D_{t}^{\bar{q}} \phi_{i j}(x, t)$, where $j=1$, $\ldots, n,|\bar{q}| \leqslant|q| \leqslant m$. The existence of $D_{t}^{\bar{q}} a_{i}(x, t)$ and $D_{t}^{\bar{q}} \phi_{i j}(x, t)(i=1, \ldots$, $k, j=1, \ldots, n)$ as well as the existence of $D_{t}^{q} b(x, t)$ is ensured by $2^{\circ}$ and $4^{\circ}$. Assumptions $1^{\circ}$ and $3^{\circ}$ guarantee that these functions are functions of class $C^{\infty}$ in $R^{n}$ for every fixed $t \in \Omega$. Therefore the coefficients $c_{p i}(x, t)$ are functions of class $C^{\infty}$ in $R^{n}$ for every fixed $t \in \Omega$.

Putting $t=\alpha$ in (5.8), and taking into account assumption $7^{\circ}$, one obtains

$$
\sum_{|p|<|q|}\left(\widetilde{c_{p}}(x) D^{p} f(x), \psi(x)\right)_{x}=(b(x, \alpha), \psi(x))_{x}
$$

for each $\psi \in D$ and for $|q| \leqslant m$, with

$$
\tilde{c}_{p}(x)=\sum_{i=1}^{k} c_{p i}(x, \alpha) .
$$

Equation (5.9) can be written shortly as

$$
\sum_{|p| \leqslant|q|} \tilde{c}_{p}(x) D^{p} f(x)=b(x, \alpha) \quad(|q| \leqslant m) .
$$

Equation (5.10) is a distributional differential equation with a $C^{\infty}$ forcing term and with $C^{\infty}$ coefficients. It follows from our proof that equation (5.10) has the same form as the equation which can be obtained by formal differentiation of equation (1.1) and evaluation for $t=\alpha$. Equation (5.10) is of constant strength, hypoelliptic at an $x_{0}$ if and only if the equation

$$
\sum_{|p|<|q|} \tilde{c}_{p}(x) D^{p} f(x)=0 \quad(|q| \leqslant m)
$$

has the same properties.

Since equation (5.11) and equation (5.1) coincide, assumption $8^{\circ}$ guarantees that there exists an $r$-tuple $q(|q| \leqslant m)$ such that (5.11), and consequently also (5.10), is of constant strength, hypoelliptic at an $x_{0}$. Therefore, applying Theorem 3.6, one concludes that every distribution $f$ satisfying equation (5.10) is a function of class $C^{\infty}$, i.e., that there exists a function $\widetilde{f} \in C^{\infty}$ such that

$$
(f, \psi)=\int_{R^{n}} \tilde{f}(x) \psi(x) d x \text { for each } \psi \in \mathcal{D} .
$$

Hence it follows that every locally integrable function $f$ which is identified with the distribution $f$ by equality (5.2) has to be equal almost everywhere to a function of class $C^{\infty}$. 
This finishes the proof.

6. Simple regularity criteria. The regularity criteria given by the author in [22] concern the linear functional equations of the form

$$
\sum_{i=1}^{m} a_{i}(x, t) f\left(x+\phi_{i}(t)\right)=f(x) .
$$

Applying Theorem 5.1 one can obtain analogous simple regularity criteria for the continuous and locally integrable solutions $f$ of nonlinear functional equations

$$
\sum_{i=1}^{k} a_{i}(x, t) f\left(x+\phi_{i}(t)\right)=F\left(x, f\left(\lambda_{1}(x)\right), \ldots, f\left(\lambda_{s}(x)\right)\right)+b(x, t) .
$$

The proofs can be done by showing that the assumptions concerning the functions $a_{i}$ and $\phi_{i}$ guarantee that condition $8^{\circ}$ of Theorem 5.1 is satisfied. Since condition $8^{\circ}$ does not depend on $F$ and on $b$ it is sufficient to quote [22] which investigated this condition in connection with linear functional equations (6.1). This way one obtains almost immediately the following theorems:

THEOREM 6.1. Suppose that

$1^{\circ} a_{i}(x, t) \in C^{\infty}$ and $b(x, t) \in C^{\infty}$ in $R^{n}$ for every fixed $t$ from an open interval $\Delta \subset R, i=1, \ldots, k$,

$2^{\circ} \quad a_{i}(x, t) \in C^{2}$ and $b(x, t) \in C^{2}$ in $R^{n} \times \Delta, i=1, \ldots, k$,

$3^{\circ} F\left(x, u_{1}, \ldots, u_{s}\right)$ is continuous in $R^{n+s}$,

$4^{\circ} \lambda_{j}(x)=\left(\lambda_{j 1}(x), \ldots, \lambda_{j n}(x)\right)$ are continuous in $R^{n}, j=1, \ldots, s$,

$5^{\circ} \phi_{i}(t)=\left(\phi_{i 1}(t), \ldots, \phi_{i n}(t)\right) \in C^{2}$ in $\Delta, i=1, \ldots, k$,

$6^{\circ}$ there exists an $\alpha \in \Delta$ such that $\phi_{i}(\alpha)=0$ and $a_{i}(x, \alpha)>0$ for $i=1$, $\ldots, k$ and for all $x \in R^{n}$.

If the vectors $\phi_{i}^{\prime}=\phi_{i}^{\prime}(\alpha)=\left(\phi_{i 1}^{\prime}(\alpha), \ldots, \phi_{i n}^{\prime}(\alpha)\right)(i=1, \ldots, k)$ span the space $R^{n}$, then every continuous solution $f: R^{n} \rightarrow R$ of equation (6.2) is a function of class $C^{\infty}$ and every locally integrable solution $f$ of this equation is equal almost everywhere to a function of class $C^{\infty}$.

THEOREM 6.2. Suppose that

$1^{\circ} b(x, t) \in C^{\infty}$ in $R^{n}$ for every fixed $t$ from an open interval $\Delta \subset R$,

$2^{\circ} b(x, t) \in C^{4}$ in $R^{n} \times \Delta$,

$3^{\circ} F\left(x, u_{1}, \ldots, u_{s}\right)$ is continuous in $R^{n+s}$,

$4^{\circ} \quad \lambda_{j}(x)=\left(\lambda_{j 1}(x), \ldots, \lambda_{i n}(x)\right)$ are continuous in $R^{n}, j=1, \ldots, s$,

$5^{\circ} \phi_{i}(t)=\left(\phi_{i 1}(t), \ldots, \phi_{i n}(t)\right) \in C^{4}$ in $\Delta, i=1, \ldots, k$,

$6^{\circ}$ there exists an $\alpha \in \Delta$ such that $\phi_{i}(\alpha)=0$ for $i=1, \ldots, k$,

$7^{\circ}$ the vectors $\dot{\phi}_{i}^{\prime}=\phi_{i}^{\prime}(\alpha)$ and $\stackrel{\alpha}{\phi}_{i}^{\prime \prime}=\phi_{i}^{\prime \prime}(\alpha)(i=1, \ldots, k)$ span the space $R^{n}$,

$8^{\circ} \Sigma_{i=1}^{k} \mu_{i}\left(\stackrel{\alpha}{\phi}_{i}^{\prime} \cdot \xi\right)\left({ }_{\phi}^{\prime \prime \prime} \cdot \xi\right) \equiv 0$ for all $\xi \in R^{n}$ 
Then every continuous solution $f: R^{n} \rightarrow R$ of the equation

$$
\sum_{i=1}^{k} \mu_{i} f\left(x+\phi_{i}(t)\right)=F\left(x, f\left(\lambda_{1}(x)\right), \ldots, f\left(\lambda_{s}(x)\right)\right)+b(x, t),
$$

where $\mu_{i}>0$ for $i=1, \ldots, k$, is a function of class $C^{\infty}$ and every locally integrable solution $f$ of this equation is equal almost everywhere to a function of class $C^{\infty}$.

To prove Theorems 6.1 and 6.2 it is enough to notice that their assumptions imply that the assumptions $1^{\circ}-7^{\circ}$ of Theorem 5.1 are satisfied and that the assumptions concerning $a_{i}$ (or $\mu_{i}$ ) and $\phi_{i}$ are the same as in Theorem 3 (or in Theorem 7) of [22].

In fact, it was shown in [22] (see p. 172) that, under the assumptions of Theorem 6.1, the equation

$$
\left.\frac{\partial^{2}}{\partial t^{2}}\left(\sum_{i=1}^{k} a_{i}(x, t) f\left(x+\phi_{i}(t)\right)\right)\right|_{t=\alpha}=0
$$

is elliptic. In view of Theorems 3.7.A and 3.7.B, condition $8^{\circ}$ of Theorem 5.1 is satisfied.

The paper [22, pp. 175-186], shows that if the vectors ${ }_{i}^{\prime}(i=1, \ldots, k)$ do not span $R^{n}$ but $\stackrel{\alpha}{\phi}_{i}^{\prime}$ and ${ }_{\phi}^{\prime \prime \prime}(i=1, \ldots, k)$ do, and if assumptions $5^{\circ}, 6^{\circ}$ and $8^{\circ}$ of Theorem 6.2 are satisfied, then the equation

$$
\left.\frac{\partial^{2}}{\partial t^{4}}\left(\sum_{i=1}^{k} \mu_{i} f\left(x+\phi_{i}(t)\right)\right)\right|_{t=\alpha}=0
$$

obtained by formal differentiation of (6.3), is hypoelliptic. Since every linear partial differential equation with constant coefficients is of constant strength, assumption $8^{\circ}$ of Theorem 5.1 is satisfied.

Applying Theorem 5.1 as a regularity criterion, one has to verify whether condition $8^{\circ}$ holds in the case considered. This often requires very long calculations. Theorems 6.1 and 6.2 allow one to obtain the same results almost without calculations. This way of finding continuous solutions can be entirely reduced to finding differentiable solutions which is considerably easier to do.

EXAMPLE 6.1. The equation

$$
\begin{aligned}
f(v-t, w)+ & f(v+t, w)+f(v, w-t)+f(v, w+t) \\
= & 4 f(v, w)+2[f(v, w)]^{2}+[f(v+w, v-w)]^{2} \\
& -[f(v, v)]^{2}-[f(w, w)]^{2}
\end{aligned}
$$

where $t, v, w \in R$, satisfies the assumptions of Theorem 6.1 with 


$$
\begin{gathered}
\phi_{1}: t \rightarrow(-t, 0), \phi_{2}: t \rightarrow(t, 0), \phi_{3}: t \rightarrow(0,-t), \\
\phi_{4}: t \rightarrow(0, t), \alpha=0, \\
F:\left(v, w, u_{1}, u_{2}, u_{3}, u_{4}\right) \rightarrow 4 u_{1}+2 u_{1}^{2}+u_{2}^{2}-u_{3}^{2}-u_{4}^{2}, \\
\lambda_{1}:(v, w) \rightarrow(v, w), \lambda_{2}:(v, w) \rightarrow(v+w, v-w), \lambda_{3}:(v, w) \rightarrow(v, v), \\
\lambda_{4}:(v, w) \rightarrow(w, w), b:(v, w, t) \rightarrow 0 .
\end{gathered}
$$

Therefore all the continuous solutions $f$ of (6.4) are functions of class $C^{\infty}$ and all the locally integrable solutions $f$ of $(6.4)$ are equal almost everywhere to $C^{\infty}$ functions.

Differentiating (6.4) with respect to $t$ and setting next $t=0$, one obtains

$$
f_{v v}(v, w)+f_{w w}(v, w)=0
$$

and

$$
f_{v v v v}(v, w)+f_{w w w w}(v, w)=0 .
$$

Simple calculations show that the functions satisfying simultaneously (6.5) and (6.6) have the form

$$
\begin{aligned}
f(v, w)= & \tilde{\alpha}\left(v^{2}-3 w^{2}\right) v+\widetilde{\beta}\left(w^{2}-3 v^{2}\right) w+\tilde{\gamma}\left(v^{2}-w^{2}\right) v w \\
& +\widetilde{\delta}\left(v^{2}-w^{2}\right)+\alpha v w+\beta v+\gamma w+\delta,
\end{aligned}
$$

where $\tilde{\alpha}, \tilde{\beta}, \tilde{\gamma}, \tilde{\delta}, \alpha, \beta, \gamma, \delta$ are arbitrary constants.

Setting $v=w=t=0$ in (6.4) once obtains $f(0,0)=0$. Now, setting $w=t=0$ in (6.4) one concludes that $f(v, 0) \equiv 0$. Therefore the substitution $w=0$ yields $f(v,-t)+f(v, t) \equiv 0$. Setting $v=t=0$ in (6.4) one obtains

$$
f(w,-w)^{2}+2 f(0, w)^{2}-f(w, w)^{2} \equiv 0 .
$$

The last two equalities imply $f(0, w) \equiv 0$.

The conditions $f(v, 0) \equiv 0$ and $f(0, w) \equiv 0$, together with (6.7), imply that the continuous solutions of (6.4) have the form

$$
f(v, w)=\tilde{\gamma}\left(v^{2}-w^{2}\right) v w+\alpha v w,
$$

where $\tilde{\gamma}$ and $\alpha$ are constants.

Setting $t=0$ in (6.4) and substituting (6.8) into the resulting equation one concludes that $\tilde{\gamma}=0$. It is easy to see that

$$
f(v, w)=\alpha v w \text {, }
$$

where $\alpha$ is an arbitrary constant, satisfies (6.4). Our considerations, based on 
Theorem 6.1, have shown that the functions $f$ defined by (6.9) are the only continuous solutions of (6.4).

\section{THEOREM 6.3. Suppose that}

$1^{\circ} \phi_{i}(t)=\left(\phi_{i 1}(t), \ldots, \phi_{i n}(t)\right) \in C^{2}$ in an open interval $\Delta \subset R, i=1$, $\ldots, k$,

$2^{\circ}$ there exists an $\alpha \in \Delta$ such that $\phi_{i}(\alpha)=0$ and $\phi_{i}^{\prime}(\alpha) \neq 0$ for $i=1$, ..., $k$.

If the equation

$$
\sum_{i=1}^{k} \mu_{i} f\left(x+\phi_{i}(t)\right)=f(x)
$$

where $\Sigma_{i=1}^{k} \mu_{i}=1$ and $\mu_{i}>0$ for $i=1, \ldots, k$, is satisfied by all the polynomials

$$
p: x \rightarrow \sum_{j, l=1 ; j \neq l}^{n} p_{j l} x_{j} x_{l},
$$

every continuous solution $f: R^{n} \rightarrow R$ of $(6.10)$ is a function of class $C^{\infty}$ and every locally integrable solution $f$ is equal to a $C^{\infty}$ solution almost everywhere.

Proof. Substituting (6.11) into (6.10) one concludes that

$$
\sum_{i=1}^{k} \mu_{i} \phi_{i j}(t) \phi_{i l}(t) \equiv 0
$$

for $j=1, \ldots, k, l=1, \ldots, k, j \neq l$.

Differentiating (6.12) twice with respect to $t$ and setting next $t=\alpha$, one obtains

$$
\sum_{i=1}^{k} \mu_{i} \phi_{i j}^{\prime}(\alpha) \phi_{i l}^{\prime}(\alpha)=0
$$

for $j=1, \ldots, k, l=1, \ldots, k, j \neq l$.

Simple calculations show that the equation

$$
\left.\frac{\partial^{2}}{\partial t^{2}}\left(\sum_{i=1}^{k} \mu_{i} f\left(x+\phi_{i}(t)\right)\right)\right|_{t=\alpha}=0,
$$

obtained by formal differentiation of (6.10), can be written as

$$
\sum_{j, l=1}^{n} \sum_{i=1}^{k} \mu_{i} \phi_{i j}^{\prime}(\alpha) \phi_{i l}^{\prime}(\alpha) f_{x_{j} x_{l}}(x)+\sum_{j=1}^{n} \sum_{i=1}^{k} \mu_{i} \phi_{i j}^{\prime \prime}(\alpha) f_{x_{j}}(x)=0,
$$

i.e., in view of (6.13),

$$
\sum_{j=1}^{n} \sum_{i=1}^{k} \mu_{i}\left[\phi_{i j}^{\prime}(\alpha)\right]^{2} f_{x_{j} x_{j}}(x)+\sum_{j=1}^{n} \sum_{i=1}^{k} \mu_{i} \phi_{i j}^{\prime \prime}(\alpha) f_{x_{j}}(x)=0 .
$$


Since $\mu_{i}>0$ and $\phi_{i}^{\prime}(\alpha) \neq(0, \ldots, 0)$ for $i=1, \ldots, k,(6.15)$ is an elliptic differential equation with constant coefficients. This means that (6.14) satisfies assumption $8^{\circ}$ of Theorem 5.1. All the other assumptions of Theorem 5.1 are obviously satisfied, and therefore every continuous solution $f$ of equation (6.10) is a function of class $C^{\infty}$ and every locally integrable solution $f$ of this equation is equal almost everywhere to a function of class $C^{\infty}$. Now, the fact that every locally integrable solution $f$ of (6.10) is equal almost everywhere to a $C^{\infty}$ solution of this equation follows from Lemma 2 of [21].

In analogy to Theorem 6.1 one can prove the following, more general, but also easily applicable, theorem.

\section{THEOREM 6.4. Suppose that}

$1^{\circ} a_{i}(x, t) \in C^{\infty}$ and $b(x, t) \in C^{\infty}$ in $R^{n}$ for every fixed $t$ from an open interval $\Delta \subset R, i=1, \ldots, k$,

$2^{\circ} a_{i}(x, t) \in C^{m}$ and $b(x, t) \in C^{m}$ in $R^{n} \times \Delta, i=1, \ldots, k$,

$3^{\circ}$ the mappings $x \mapsto y=\phi_{i}(x, t)$ are diffeomorphisms in $R^{n}$ for every fixed $t \in \Delta, i=1, \ldots, k$,

$4^{\circ} \phi_{i}(x, t) \in C^{m}$ and its inverse $\phi_{i}^{-1}(y, t) \in C^{m}$ in $R^{n} \times \Delta, i=1, \ldots, k$,

$5^{\circ} F\left(x, u_{1}, \ldots, u_{s}\right)$ is continuous in $R^{n+s}$,

$6^{\circ} \lambda_{j}(x)$ are continuous in $R^{n}, j=1, \ldots, s$,

$7^{\circ}$ there exists an $\alpha \in \Delta$ such that $\phi_{i}(x, \alpha) \equiv x$ and $a_{i}(x, \alpha)>0$ for all $x \in R^{n}, i=1, \ldots, k$.

If, for every fixed $x \in R^{n}$, the vectors

$$
\begin{aligned}
\stackrel{\alpha}{\chi}_{i}(x) & =\left.\frac{\partial}{\partial t} \phi_{i}(x, t)\right|_{t=\alpha}=\left.\left(\frac{\partial}{\partial t} \phi_{i 1}(x, t), \ldots, \frac{\partial}{\partial t} \phi_{i n}(x, t)\right)\right|_{t=\alpha} \\
& =\left(\stackrel{\alpha}{\chi}_{i 1}(x), \ldots, \stackrel{\alpha}{X n}_{i n}(x)\right) \quad(i=1, \ldots, k)
\end{aligned}
$$

span the space $R^{n}$, every continuous solution $f: R^{n} \rightarrow R$ of the equation

$$
\sum_{i=1}^{k} a_{i}(x, t) f\left(\phi_{i}(x, t)\right)=F\left(x, f\left(\lambda_{1}(x)\right), \ldots, f\left(\lambda_{s}(x)\right)\right)+b(x, t),
$$

where $n>1, r=1$, is a function of class $C^{\infty}$ and every locally integrable solution $f$ of this equation is equal almost everywhere to a $C^{\infty}$ function.

Proof. Notice that assumptions $1^{\circ}-6^{\circ}$ of this theorem coincide with assumptions $1^{\circ}-6^{\circ}$ of Theorem 5.1 and that assumption $7^{\circ}$ includes assumption $7^{\circ}$ of Theorem 5.1. Thus it also remains to prove that assumption $8^{\circ}$ of Theorem 5.1 is satisfied.

The principal part of the equation

$$
\left.\frac{\partial^{2}}{\partial t^{2}}\left(\sum_{i=1}^{k} a_{i}(x, t) f\left(\phi_{i}(x, t)\right)\right)\right|_{t=\alpha}=0
$$


can be written as

$$
\sum_{i=1}^{k} a_{i}(x, \alpha) \sum_{j, l=1}^{n} \stackrel{\alpha}{\chi}_{i j}(x){\stackrel{\alpha}{\chi_{i l}}}(x) f_{x_{j} x_{l}}(x)
$$

The corresponding quadratic form

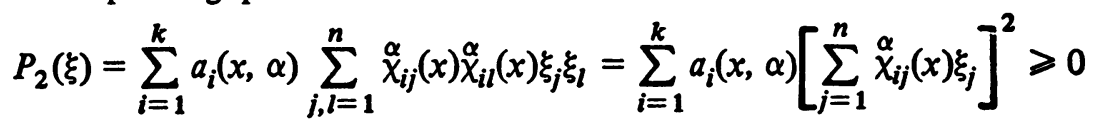

since, in view of $7^{\circ}, a_{i}(x, \alpha)>0$ for $x \in R^{n}, i=1, \ldots, k$.

To prove that $P_{2}(\xi)>0$ for all $\xi \neq 0$ notice that $P_{2}(\xi)=0$ if and only if

$$
\sum_{i=1}^{n} \stackrel{\alpha}{\chi}_{i j}(x) \xi_{j}=0 \quad \text { for } i=1, \ldots, k .
$$

If equalities (6.18) hold for a $\xi \neq 0, \xi$ is orthogonal to all $\stackrel{\alpha}{\chi}_{i}(x)(i=1$, $\ldots, k)$. However, this contradicts the assumption that the vectors $\chi_{i}^{\alpha}(x)(i=1$, $\ldots, k)$ span $R^{n}$. Therefore $P_{2}(\xi)>0$ for all $\xi \neq 0$, i.e. (6.17) is an elliptic differential equation.

By Theorems 3.7.A and 3.7.B, condition $8^{\circ}$ of Theorem 5.1 is satisfied.

\section{Final remarks.}

REMARK 7.1. Theorems 3.4 and 3.7.C allow one to obtain a proof of Theorem 6.1 without using Theorem 5.1. Similarly, Theorems 3.4 and 3.8 allow one to prove Theorem 6.2 without using Theorem 5.1. However, these methods (see H. Swiatak [19]) are not applicable in the case $\phi_{i}(x, t) \neq x+\phi_{i}(t)$.

REMARK 7.2. Theorem 5.1 is a general, but sometimes difficult to apply, regularity criterion. However, it allows one to approach the problem of finding simple regularity criteria in the case $\phi_{i}(x, t) \neq x+\phi_{i}(t)$. Theorem 6.4 exemplifies such a situation.

REMARK 7.3. Theorem 6.4 could be formulated also for $r>1$.

REMARK 7.4. A theorem like 5.1 was announced by the author in [20] and, together with additional results, in [23]. The paper [23] (see also [24]) announces, without a detailed proof, Theorem 5.1 and some more general regularity criteria which follow from this theorem. (Adding not very restrictive assumptions one can prove that all the locally integrable solutions $f$ of (1.1) are equal almost everywhere to $C^{\infty}$ solutions of this equation. The fact that every locally integrable solution $f$ of $(1.1)$ is a $C^{\infty}$ function was proved by the author under a rather strong assumption $r \geqslant n$. The results of [17], concerning mean value equations, show that such an assumption is not necessary.)

REMARK 7.5. Lemma 4.1 can be used to prove an analogue of Theorem 5.1 for functional differential equations. The results of this type were presented by the author during the 9th International Symposium on Functional Equations, Roma-Procchio, Italy, September 1971 (see [26]). 


\section{REFERENCES}

1. J. Aczel, Lectures on functional equations and their applications, Math. in Sci. and Engineering, vol. 19, Academic Press, New York and London, 1966. MR 34 \#8020.

2. J. Aczél, H. Haruki, M. A. McKiernan and G. N. SakoviX, General and regular solutions of functional equations characterizing harmonic polynomials, Aequationes Math. 1 (1968), 37-53. MR 43 \#5193.

3. J. A. Baker, An analogue of the wave equation and certain related functional equations, Canad. Math. Bull. 12 (1969), 837-846. MR 40 \#7663.

4. G. Choquet and J. Deny, Sur quelques propriétés de moyenne caractéristiques des fonctions harmoniques et polyharmoniques, Bull. Soc. Math. France 72 (1944), 118-140. MR 7, 161.

5. I. Feny४, Über eine Lösungsmethode gewisser Funktionalgleichungen, Acta Math. Acad. Sci. Hungar. 7 (1956), 383-396. MR 19, 152.

6. - Über eine Funktionalgleichung, Math. Nachr. 31 (1966), 103-109. MR 33 \#4509.

7. - Bemerkungen zur Funktionalgleichung $f(x+y)+f(x-y)+a f(x)=$ $2 g(x) h(y)$, Glasnik Mat. Ser. III 1 (21) (1966), 69-73. MR 35 \#1995.

8. L. Flatto, Functions with a mean value property, J. Math. Mech. 10 (1961), 1118. MR 25 \#212.

9. - Functions with a mean value property. II, Amer. J. Math. 85 (1963), 248-270. MR 28 \#1314.

10. Partial differential equations and difference equations, Proc. Amer. Math. Soc. 16 (1965), 858-863. MR 31 \#6073.

11. On polynomials characterized by a certain mean value property, Proc. Amer. Math. Soc. 17 (1966), 598-601. MR 33 \#4217.

12. A. Friedman and $W$. Littman, Functions satisfying the mean value property, Trans. Amer. Math. Soc. 102 (1962), 167-180. MR 27 \#1612.

13. A. M. Garsia, $A$ note on the mean value property, Trans. Amer. Math. Soc. 102 (1962), 181-186. MR 27 \#1613.

14. A. M. Garsia and E. Rodemich, On functions satisfying the mean value property with respect to a product measure, Proc. Amer. Math. Soc. 17 (1966), 592-594. MR 33 \#2793.

15. L. Hơrmander, Linear partial differential operators, Die Grundlehren der Math. Wissenschaften, Band 116, Academic Press, New York; Springer-Verlag, Berlin, 1963.

MR 28 \#4221.

16. B. Lawruk and $\mathrm{H}$. Światak, On functions satisfying a generalized mean value equation, Aequationes Math. 11 (1) (1974), 1-10. MR 49 \#9460.

17. M. A. McKiernan, Boundedness on a set of positive measure and the mean value property characterizes polynomials on a space $V^{n}$, Aequationes Math. 4 (1970), 31-36. MR 45 \#762.

18. L. Schwartz, Théorie des distributions, Publ. Inst. Math. Univ. Strasbourg, no. IX-X, Hermann, Paris, 1966. MR 35 \#730.

19. H. Swiatak, On the regularity of the distributional and continuous solutions of the functional equations $\sum_{i=1}^{k} a_{i}(x, t) f\left(x+\phi_{i}(t)\right)=b(x, t)$, Aequationes Math. 1 (1968), 6-19. MR 43 \#5196.

20. On the regularity of the solutions of a class of functional equations, Notices Amer. Math. Soc. 16 (1969), 512. Abstract \#664-41.

21. On the regularity of the locally integrable solutions of the functional equations $\Sigma_{i=1}^{k} a_{i}(x, t) f\left(x+\phi_{i}(t)\right)=b(x, t)$, Aequationes Math. 4 (1970), 291-296.

22. , Criteria for the regularity of continuous and locally integrable solutions of a class of linear functional equations, Aequationes Math. 6 (1971), 170-187. MR 45 \#763.

23. - On certain regularity problems for solutions of functional equations, Bull. Acad. Polon. Sci. Sér. Sci. Math. Astronom. Phys. 19 (1971), 209-212. MR 45 \#7335. 
24. H. Swiatak, On the regularity of the continuous and locally integrable solutions of a class of functional equations, Aequationes Math. 4 (1970), Reports of Meetings, 233.

25. - On generalized mean value equations, Aequationes Math. 7 (1972), Reports of Meetings, 262-263.

26. - Regularity problems for the solutions of functional differential equations, Aequationes Math. 8 (1972), Reports of Meetings, 146-147.

27. F. Trèves, Linear partial differential equations with constant coefficients:

Existence, approximation and regularity of solutions, Math. and Appl., vol. 6, Gordon and Breach, New York, 1966. MR 37 \#557.

28. L. Zalcman, Mean values and differential equations, Israel J. Math. 14 (1973), 339-352. MR 49 \#613.

DÉPARTEMENT DE MATHÉMATIQUES, UNIVERSITÉ DE MONTRÉAL, MONTRÉAL, QUÉBEC, CANADA 